\title{
COMPARISION BETWEEN OPEN AND LAPAROSCOPIC SPLENEC- TOMY FOR HAEMATOLOGICAL DISORDER
}

\author{
Sajad Geelani ${ }^{1}$, Sahil Sandal ${ }^{2}$, Mubashir Shah ${ }^{3}$, Sameer Naqash ${ }^{4}$, Syed Mudasir \\ Qadri ${ }^{5}$, Javid Rasool ${ }^{6}$, Subuh Parvez Khan *7.
}

${ }^{1}$ Additional Professor, Department of Clinical Haematology, Sher e Kashmir Institute of Medical Sciences, Srinagar, J\&K, India.

${ }^{2}$ MS General Surgery, Sher e Kashmir Institute of Medical Sciences,Srinagar, J\&K, India.

${ }^{3}$ Additional Professor, Department of general and minimal invasive surgery, Sher e Kashmir Institute of Medical Sciences, Srinagar, J\&K, India.

${ }^{4}$ Professor, Department of general and minimal invasive surgery, Sher e Kashmir Institute of Medical Sciences, Srinagar, J\&K, India.

${ }^{5}$ Associate Professor, Department of Internal Medicine, Sher e Kashmir Institute of Medical Sciences, Srinagar, J\&K, India.

${ }^{6}$ Professor and Head of department of Clinical Haematology Sher e Kashmir Institute of Medical Sciences, Srinagar, J\&K, India.

${ }^{* 7}$ Senior Resident, Department of Haematopathology, Sher e Kashmir Institute of Medical Sciences, Srinagar, J\&K India.

\section{ABSTRACT}

\begin{abstract}
Introduction: Splenectomy addresses the role of the spleen in hematological disorders particularly those of cellular sequestration and destruction and antibody production. Splenectomy is indicated in specific cases of anemia.

Material and methods: The study was conducted in the department of General and Minimal Invasive Surgery in collaboration with the department of Clinical Hematology, Sher-I-Kashmir Institute of Medical Sciences, Srinagar from Jan 2012 to Jan 2016.The study included 33 patients The patients were studied in regards to the variables1.clinical and demographic information. 2.surgical findings.3.preoperative and post operative haematological profile.4.immediate and late complications.5.hospital stay6.persistence of disease.

Result: In our study, out of 33 patients, laparascopic splenectomy(LS) was performed on 19 patients(57.6\%) and 14 underwent open splenectomy(OS).. Mean age was 28.89 years in LS group.and 36 years in OS group. Male female ratio was $4 / 15$ in LS and $3 / 11$ in OS. Mean operation time was 107.5 minutes in LS and 80.7 minutes in OS(p value <.001). Mean intraoperative blood loss was significantly lower for LS vs OS $(76.43 \mathrm{~mL}$ vs $133.57 \mathrm{~mL} ; P=$ .003). There was no statistical significant difference in complications between the two groups. Also, There was no significant difference between LS and OS in terms of remission rates.

Conclusion: Laparoscopic splenectomy is a safe and effective procedure in experienced hands. It is superior with regard to hospital stay, operative blood loss, postoperative pain and cosmesis as compared to Open Splenectomy.

KEY WORDS: Splenectony, Open,Laparascopic, Haematology.
\end{abstract}

Address for correspondence: Dr.Subuh Parvez Khan, Senior Resident, Department of Haematopathology, Sher e Kashmir Institute of Medical Sciences, Srinagar, J\&K India.

E-Mail:khansubuh@gmail.com

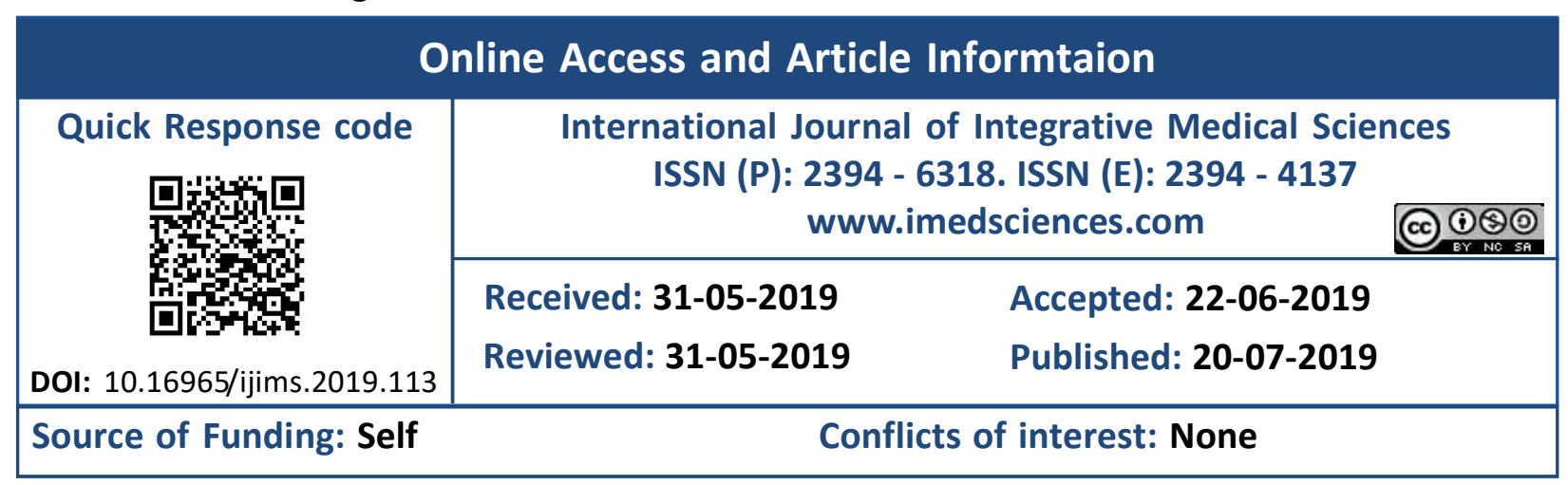




\section{INTRODUCTION}

Splenectomy addresses the role of the spleen in hematological disorders particularly those of cellular sequestration and destruction and antibody production.Splenectomy is indicated in specific cases of anemia. The two major categories of anemia that benefit from splenectomy are 1. Intracellular defects including membrane abnormalities, enzyme defects and hameoglobinpathies. 2. Extracellular defects, particularly autoimmune hemolytic anemia.

Splenectomy is effective in reducing hemolysis associated with hereditary spherocytosis. However, splenectomy in these cases is reserved for older patients because of increased risk of sepsis in children younger than 6 years. Splenectomy is considered in immune hemolytic anemias in patients who fail to corticosteroid treatment.In patients with thalassemia, though splenectomy doesn't influence the basic haematological disorder, it may eliminate or reduce the haemolytic process. Splenectomy is of benefit in only a few patients of sickle cell anemia in whom excessive splenic sequestration of RBCs, WBCs and platelets can be demonstrated. In Idiopathic thrombocytopenic purpura, corticosteroids are the first line of therapy. Splenectomy should be performed in patients who fail to respond to treatment within 6 months, who respond to medical treatment but do not tolerate its side effects, or who develop intracranial bleeding or profound GI bleeding and do not respond to intensive medical treatment [1]. The laparascopic approach to splenectomy is well suited in ITP because of normal size of spleen.

AIM: To compare open and laparascopic splenectomy in terms of expected blood loss, intraoperative time, morbidity and surgical complication and response in terms of blood parameters.

\section{MATERIALS AND METHODS}

The study was conducted in the department of General and Minimal Invasive Surgery in collaboration with the department of Clinical Hematology, Sher-I-Kashmir Institute of Medical Sciences, Srinagar from Jan 2012 to Jan 2016.The study included 33 patients

Inclusion criteria: 1.Age $>13$ years, 2.Confirmed haematological disorder.
Exclusion criteria: 1.Splenctomy for malignant haematological disorders.

The patients were studied in regards to the variables 1. clinical and demographic information. 2.surgical findings. 3. preoperative and post operative haematological profile. 4. immediate and late complications. 5 . hospital stay 6 . persistence of disease.

The study was given consent by the institutional ethical committee.

\section{RESULTS}

In our study, out of 33 patients, laparascopic splenectomy(LS) was performed on 19 patients(57.6\%) and 14 underwent open splenectomy(OS). Age ranged between 13 and 71 years in LS group. Mean age was 28.89 years in LS group. In OS group, age ranged between 17 and 56 years with mean of 36 years. Male female ratio was $4 / 15$ in LS and $3 / 11$ in OS. OS was performed in 14 patients. 11 patients had ITP, 2 patients had hypersplenism and 1 patient had autoimmune hemolytic anemia.

LS was performed in 19 patients. 15 had chronic ITP , 3 had AIHA and 1 had aplastic anaemia. LS was completed successfully in 84.3 percent. Mean operation time was 107.5 minutes in LS and 80.7 minutes in OS( $p$ value <.001). Mean intraoperative blood loss was significantly lower for LS vs OS (76.43 mL vs $133.57 \mathrm{~mL} ; P=.003$ ). Mean hospital stay was 3.93 days in LS patients. The hospital stay was 6.00 days in OS patients. Significant intraoperative bleeding, which couldnot be controlled laparoscopically, occured in 3 patients in LS. The postoperative complications in Open group included wound infections in 2 patientsIn LS, Subphrenic collection developed in 1 patient and Atelactasis in 1 patient. There was no postoperative pancreatic fistula formation in our study. However on comparing the two groups, there was no statistical significant difference in complications between the two groups.

Accessory spleens were found intraoperatively in $3(15.7 \%)$ patients undergoing LS and were extracted. In OS, accessory spleen was found in 1 patient (7\%). complete remission was seen in $94 \%$ of the patients in LS and $91 \%$ of the patients in OS $(p>0.05)$.Thus there is no significant difference between LS and OS in terms of 
remission rates.

\section{DISCUSSION}

In our study, age ranged between 13 and 71 years in LS group. Mean age was 28.89 years in LS group. In OS group, age ranged between 17 and 56 years with mean of 36 years. In study conducted by N. Jankulovski et al [2] 2013, mean age was 37.3 years in LS and 39.7 years in OS. The age in both groups was similar (LS $40.6 \pm$ 18.5 vs. OS $38.6 \pm 17.5$ ) in study conducted by Sapucahy MV et al [3] in 2003. In study conducted by Yikun Qu et al [4] 2014, mean age was 37.9 years in LS and 35.6 years in OS. The possible reason that why the mean age was less in our study may be the early referral for splenectomy due to the cost of conservative treatment.

In our study, male female ratio was $4 / 15$ in LS and $3 / 11$ in OS. In a study conducted by Yikun Qu et al [4] 2014, male female ratio was 18/14 in LS and $24 / 17$ in OS. In our study most of patients had ITP as underlying disorders that is why our study had increased female ratio as ITP is more common in females [5].

OS was performed in 14 patients. 11 patients had ITP, 2 patients had hypersplenism and 1 patient had autoimmune hemolytic anemia.LS was performed in 19 patients. 15 had chronic ITP , 3 had AIHA and 1 had aplastic anaemia.

LS was completed successfully in $84.3 \%$. 3 patients were converted to open surgery with conversion rate of $15.7 \%$. This is consistent with the study conducted by Sapucahy MV et al [3] in 2013, 4 patients out of 30 were converted to open with a conversion rate of $13.3 \%$. In a study conducted by Yikun Qu et al [4], LS was converted to OS in 4 of 32 patients (12.5\%). The reason for conversion was excessive bleeding that could not be controlled by routine laparoscopic hemostatic measures.

In our study, mean operation time was 107.5 minutes in LS and 80.7 minutes in OS. During the study, the mean operative time for LS showed a decreasing trend. Mean operative time was 115.5 min in the initial 9 cases of LS which got reduced to $95.5 \mathrm{~min}$ in the later 10 cases of LS. LS were started in our centre since 2012. Operation time was longer in our early experience $(115.5 \mathrm{~min})$. The mean time dropped (95.5min) as the experience of the surgeon increased. The operative time was longer where the spleen was removed piecemeal from the port site after putting it inside sterile polythene bag within the abdomen. Our study is comparable to study conducted by Park $A$ el al [6] (145.1 vs 77.3 minutes; $P<.001$ ). In a study conducted by Yikun Qu et al [4] in, mean operation time were $160 \mathrm{~min}$ in LS and $98 \mathrm{~min}$ in OS. Mean intraoperative blood loss was significantly lower for LS vs OS (76.43 mL vs $133.57 \mathrm{~mL} P=.003$ ) in our study. This is because of the advantage of magnification in laparascopy and the use of gadgets like harmonic scalpel and bipolar cautery that prevent excessive bood loss. Our study is comparable to the study conducted by Park $A$ et al [6] in which mean intraoperative blood loss was significantly lower for LS vs OS $(162.3 \mathrm{~mL}$ vs $380.8 \mathrm{~mL} ; P=0.002$ ). Our study is also consistent with the study conducted by Yikun Hu et al [4] in 2014 in blood loss in LS was $100 \mathrm{ml}$ and $367 \mathrm{ml}$ in $\mathrm{OS}(\mathrm{p}<0.01)$.

Since the histopathological examination of spleen is not required for the hematological disorders, the spleen was removed in piecemeal through portsite after bagging it in 12 patients of LS. While in rest of the patients, it was removed by open incision, 2 by Pfannensteil and 2 by subcostal incision.

In our study, mean hospital stay was 3.93 days in LS patients. The hospital stay was 6.00 days in OS patients. The differences between LS and OS were statistically significant in favour of LS which is comparable with the study conducted by Franciosi C et al [7] 2000 in which mean postoperative hospital stay was 4.1 days for LS, compared with 8.1 days for OS( $P<0.001)$.Our study is also comparable to the study conducted by Nyilas A et $\mathrm{al}^{8}$ in 2013 in which hospital stay was shorter ( 5 in LS vs. 8 days in OS, $\leq 0.001$ ). Hospital stay was 8.9 days in LS and 11.7 days in OS in study led by Yikun Qu et al [4] in 2014( $p=0.01)$.

Complications: Significant intraoperative bleeding, which could not be controlled laparoscopically, occured in 3 patients in LS (15\%) leading to conversion. In a study conducted by Park $A$ et al [6] in 1999, intraoperative bleeding occured in 15 patients out of 147(10.2\%). 
The postoperative complications in Open group included wound infections in 2 patients (14\%), Subphrenic collection in 1 patient, Atelectasis in 2 patients and Incisional hernia in 2 patients. In LS, Subphrenic collection developed in 1 patient and Atelactasis in 1 patient. There was no postoperative pancreatic fistula formation in our study. However on comparing the two groups, there was no statistical significant difference in complications between the two groups. Our study is comparable to the study conducted by Bulus $\mathrm{H}$ et al [9] in 2013 where incidence of postoperative complications were more in OS but statistically insignificant(0.06).

In our study, accessory spleens were found intraoperatively in 4 patients (12.1\%) which is comparable with the study conducted by Rosen M et al [10] where they found accessory spleen in $14 \%$ of the patients. An accessory spleen was found in $16 \%$ patients in a study conducted by Delaitre B et al [11]. In our study, 3 (15.7\%) were found in LS and were extracted. In OS, accessory spleen was found in 1 patient $(7 \%)$. In a study done by Park $A$ et al [6], 22 accessory spleens (15.0\%) were identified and resected in the LS group, but only 3 were recorded in OS patients (4.8\%). The chances of detecting an accessory spleeen is more in laparoscopic approach than in open approach because of better visualization and magnification provided by laparoscope.

In our study, partial response (after 6 months) was detected in 2 patients of ITP in short-term follow-up one in each group with a partial response rate of $6 \%$ in LS and $9 \%$ in OS. Thus complete remission was seen in $94 \%$ of the patients in LS and $91 \%$ of the patients in OS ( $p>0.05$ ) which is comparable to study conducted by Yikun Qu [4] et al in 2014 in which 25 patients (86\%) in LS group and 32 (91\%) in OS group reached sustained complete response $(P=$ 0.792 ). Thus there is no significant difference between LS and OS in terms of remission rates. In conclusion, Laparoscopic splenectomy is a safe and effective procedure in experienced hands. It is superior with regard to hospital stay, operative blood loss, postoperative pain and cosmesis as compared to Open Splenectomy. There is no difference between Laparoscopic Splenectomy and Open Splenectomy in terms of hematological response in ITP. Postoperative complications are similar except that the wound related complications are more commonly seen in Open splenectomy.Though Laparoscopic Splenectomy takes more time compared to Open splenectomy, with increasing experience of the laparoscopic surgeon the time gap also decreases.

\section{REFERENCES}

[1]. Chirletti P, Cardi M, Barillari P, et al. Surgical treatment of immune thrombocytopenic purpura. World J Surg 1992;16(5):1001-1005. [PubMed: 1462608]

[2]. Nikola Jankulovski et al: LAPAROSCOPIC VERSUS OPEN SPLENECTOMY: A SINGLE CENTER ELEVEN-YEAR EXPERIENCE .Acta Clin Croat 2013;52:229-234.

[3]. Sapucahy MV et al: LAPAROSCOPIC VERSUS OPEN SPLENECTOMY IN THE MANAGEMENT OF HEMATOLOGIC DISEASES.REV. HOSP. CLÍN. FAC. MED. S. PAULO 2000;58(5):243-249.

[4]. Yikun Qu et al: Long-Term Outcomes of Laparoscopic Splenectomy Versus Open Splenectomy for Idiopathic Thrombocytopenic Purpura.Int Surg 2014;99:286-290 DOI: 10.9738/INTSURG-D-1300175.1

[5]. Sabiston: Textbook of Surgery:19 ${ }^{\text {th ed }}$ :vol2, chap 67; page1551.

[6]. Park A et al: Laparoscopic vs Open Splenectomy. 1999;134(11).

[7]. Franciosi C et al :Laparoscopic versus open splenectomy: a comparative study. Surg Laparosc Endosc Percutan Tech. 2000 Oct;10(5):291-5.

[8]. Nyilas A et al: Comparison of laparoscopic and open splenectomy. Magy Seb.2013 Feb;66(1):14-20. doi: 10.1556/MaSeb.66.2013.1.2.

[9]. Hakan Bulus et al :Outcomes of laparoscopic versus open splenectomy.J Korean Surg Soc. 2013 Jan;84(1):38-42.

[10]. Rosen $M$ et al: Outcome of laparoscopic splenectomy based on hematologic indication. Surg Endosc. 2002 Feb;16(2):272-9. Epub 2001 Nov 12.

[11]. Delaitre B et al. Laparoscopic splenectomy for hematologic diseases. Study of 275 cases. French Society of Laparoscopic Surgery. Ann Chir. 2000 Jul;125(6):522-9.

How to cite this article: Sajad Geelani, Sahil Sandal, Mubashir Shah, Sameer Naqash, Syed Mudasir Qadri, Javid Rasool, Subuh Parvez Khan. COMPARISION BETWEEN OPEN AND LAPAROSCOPIC SPLENECTOMY FOR HAEMATOLOGICAL DISORDER. Int J Intg Med Sci 2019;6(4):817-820. DOI: 10.16965/ijims.2019.113 